\title{
Antiphospholipid Syndrome Presenting as Mesenteric Vein Thrombosis and Gangrenous Small Bowel
}

\author{
Wan Ali WASR, ${ }^{a}$ Mohd Shahrir MS, ${ }^{b}$ Hussein $\mathrm{H}^{\mathrm{c}}$ \\ aDepartment of Internal Medicine Faculty of Medicine, International Islamic University Malaysia. \\ ${ }^{b}$ Consultant Rheumatologist, Department of Internal Medicine Faculty of Medicine, Universiti Kebangsaan \\ Malaysia Medical Centre \\ 'Consultant Rheumatologist, Medical Department, Hospital Putrajaya, Putrajaya
}

\begin{abstract}
A 35-year-old Jordanian gentleman presented with worsening right iliac fossa pain of three-day durations with vomiting, loose stool and intermittent fever. He later underwent laparoscopic surgery, which was subsequently converted to open laparotomy. Intraoperatively, there was thickened bowel with multiple mesenteric lymph nodes, and also thrombosis of the small bowel vein supplying the affected bowel lesion, the diagnosis which was later confirmed with the histopathological report. Resection with end-to-end anastomosis was done during the surgery. Within one-week post laparotomy, he developed another progressive abdominal pain and distension, vomiting and no bowel opening. He was sent for another exploratory laparotomy. Intraoperatively, there was a gangrenous small bowel segment measuring $130 \mathrm{~cm}$, with an intact large bowel and previous anastomotic site. Resection of $150 \mathrm{~cm}$ of the small bowel was subsequently done.
\end{abstract}

KEYWORDS: antiphospholipid syndrome, mesenteric veins, venous thrombosis

\section{INTRODUCTION}

Antiphospholipid syndrome (APS) is characterized by recurrent venous or arterial thrombosis and/or fetal losses associated with elevated levels of antibodies directed against membrane anionic phospholipids, most commonly anticardiolipin antibody $(\mathrm{aCL}) .{ }^{1}$ The actual incidence of APS in the general population worldwide is unknown. This is because the antiphospholipid antibodies are also present in one to five percent of normal individuals, especially in the elderly, and also in those with other comorbidities such as cancer, severe atherosclerosis, or chronic and/ or acute infection. ${ }^{2}$ However, this antibody is more commonly found in systemic lupus erythematosus (SLE) patients, which in a few studies have been shown that it is found in approximately $30-40 \%$ of patients with SLE. However, out of that number, only about $10 \%$ can be diagnosed to have APS. ${ }^{2}$ The presence of antiphospholipid antibodies in young, healthy patients, are also associated with thromboembolism rates of $6 \%$ to $8 \%$ reported in a few case reports.

The diagnosis of APS is made by a combination of one clinical criteria plus one laboratory criteria (Revised

Corresponding author:

Dr Mohd Shahrir Mohamed Said

Associate Professor and Consultant Rheumatologist Medical Department

Universiti Kebangsaan Malaysia Medical Centre

Jalan Yaacob Latif, Cheras

56000 Kuala Lumpur

Email: drobiwan@gmail.com
Sapporo APS Update 2006). The clinical criteria are vascular thrombosis and pregnancy events, with evidence from histopathological examination or imaging studies. The laboratory criterion is a medium to high level of anticardiolipin antibody, and beta-2 glycoprotein-I; or a positive lupus anticoagulant antibody 12 weeks apart. ${ }^{3}$

\section{CASE REPORT}

A 35-year-old Jordanian gentleman presented with worsening of right iliac fossa pain of three days duration with worsening of vomiting, loose stool and intermittent fever. The right iliac fossa pain was continuous, cramping and not relieved with analgesics. It was also not associated with food intake or body movement. There were also no urinary symptoms or no similar history before. The loose stool occurred about the same onset as the right iliac fossa pain, associated with diarrhoea. There was no history of vomiting of blood, or passing of blood per rectum. Patient also denied taking outside food or painkillers in the past. The fever was intermittent, with no chills and rigors. There was also no complaint of cough or urinary symptoms.

He also had a background history of deep-veinthrombosis in 2005, with positive lupus anticoagulant antibody. At that time, he was treated in University Malaya Medical Centre with warfarin for six months, and was not on any follow - up after he completed six months of warfarin. There was no similar history running in his family. He was also a non-smoker and a non-alcohol consumer.

Clinically, there was tenderness without any guarding over the right iliac fossa region. The serum amylase, renal profile and also liver function tests were normal. The anti-nuclear antibody (ANA) was also negative, 
with normal levels of complement levels of C3 and C4 of 102 and $15 \mathrm{mmol} / \mathrm{L}$ respectively. Lupus anticoagulant and anticardiolipin antibodies were negative, with negative hepatitis and thrombophilia screening.

Laparoscopic surgery was preceded and later converted to open laparotomy in view of intraoperative findings of gangrenous small bowel. Intraoperatively, there was thickened bowel with multiple mesenteric lymph nodes. The small bowel lesion was located 50 $\mathrm{cm}$ from the terminal ileum, with multiple mesenteric lymph node measuring $20 \mathrm{~cm}$ in length. Another lesion was located $20 \mathrm{~cm}$ from the terminal ileum similar to the first lesion, measuring $20 \mathrm{~cm}$ in length with associated thrombosis of the small bowel vein and gangrene of the affected area. There were no bowel obstructions, liver nodule or enlarged para-aortic lymph nodes. The appendix and the rest of small bowel and colon were normal. The histopathological report confirmed the findings. Resection with end-to-end anastomosis was done during the surgery.

Within one-week post laparotomy, he developed another progressive abdominal pain and distension, vomiting and no bowel opening. The signs and symptoms were suggestive of acute intestinal obstruction. He underwent another emergency exploratory laparotomy. Intraoperatively, there was a small bowel gangrene measuring $130 \mathrm{~cm}$, with an intact large bowel and previous anastomotic site. Resection of $150 \mathrm{~cm}$ of the small bowel was then done, and the 2 ends of the bowel were brought as a double barrel stoma.

He was started on low-molecular-weight heparin (subcutaneous clexane), and intravenous methylprednisolone initially, which was later converted to oral prednisolone. Low-molecular-weight heparin was later changed to lifelong oral warfarin. Later, he presented again with complications of the surgery, with short bowel syndrome. He recovered after diet modification and counselling before discharge.

\section{DISCUSSION}

Antiphospholipid syndrome (APS) or otherwise known as Hughes' Syndrome ${ }^{1}$ is a disorder which manifests itself by recurrent arterial or venous thrombosis, with persistently elevated levels of antibodies directed against membrane anionic phospholipids (e.g. anticardiolipin $[\mathrm{aCL}]$ antibody, antiphosphatidylserine) or their associated plasma proteins, predominantly beta-2 glycoprotein I (apolipoprotein $\mathrm{H}$ ), or evidence of a circulating anticoagulant. ${ }^{1}$

The diagnosis of a definite APS is made by referring to the revised Sapporo Criteria 2006, whereby it consists of at least one clinical criteria, which involves one or more episodes of vascular thrombosis and also one laboratory criteria with positive anticardiolipin $(\mathrm{aCL})$, anti-beta-2 glycoprotein I, or lupus anticoagulant antibody on at least 2 occasions at least 12 weeks apart.
The episode of vascular thrombosis must be confirmed by imaging, doppler studies, or histopathology. ${ }^{1}$

This patient fits the clinical diagnosis of antiphospholipid syndrome in view of he presented with two proven episodes of recurrent venous thrombosis, the first one involving the lower limb deep-vein-thrombosis in 2005, and the second one is the current problem which involved thrombosis of the mesenteric veins. However, he did not totally fit into the laboratory criteria, whereby there must be a positive antiphospholipid antibody at two occasions at least six weeks apart, because even though the anticardiolipin antibody was positive in 2005, the second anticardiolipin antibody screening and also lupus anticoagulant is negative. Other tests were also sent and the only positive test is the cryoglobulin antibody. Anti-beta 2 glycoprotein-1 and other antiphospholipid antibodies were not sent.

The antiphospholipid antibodies associated with APS are anticardiolipin antibodies $(\mathrm{aCL})$, lupus anticoagulants (LAs) which are immunoglobulins directed against plasma proteins (prothrombin or annexin V) and anti-beta-2 glycoprotein-I (anti-beta-2GPI) antibodies. According to a few studies, the prevalence of anti-beta-2 glycoproteins in APLS ranges from zero percent to 90 percent. ${ }^{2}$ Anti-beta-2 glycoprotein-I antibodies have higher specificity than $\mathrm{aCL}$ for thrombosis. ${ }^{2}$

There were two evaluations conducted of the proposed and revised Sapporo criteria. In the first evaluation by Kaul et al., ${ }^{3}$ whereby a retrospective analysis of 200 aPL-positive patients who met the previous diagnostic criteria for APLS (1999 Sapporo criteria), only 59 percent can be diagnosed with APLS by using the 2006 revised criteria. ${ }^{4}$ Thus, the 2006 revised criteria is likely to exclude a significant number of patients presented with APS clinically. However, the 2006 criterion is useful in defining a more homogeneous population, especially for research studies. ${ }^{4}$

This patient is likely to have APLS in view of the recurrent venous thrombosis, even though he does not fulfill the laboratory criteria. This is because the repeat lupus anticoagulant antibody test is negative, and also there might be a positive anti beta- 2 microglobulin antibody, which was not sent for investigation. There was also no history of trauma or prolonged immobilization, and we have ruled out other possibilities like hypercoagulable state and thrombophilia, which can also cause recurrent thrombosis.

Another issue in this patient is whether the APLS is primary or secondary. Primary antiphospholipid syndrome means that the APLS occurs by itself and not associated with a connective tissue disease, particularly with the spectrum of systemic lupus erythematosus. In this patient, he has primary antiphospholipid syndrome in view of negative connective tissue disease screening, namely 
anti-nuclear antibody (ANA), complement level C3/ C4, rheumatoid factor and anticardiolipin antibody IgG/lgM. There are also no other signs or symptoms suggestive of a related connective tissue disease in the patient. In the European Multicenter Study of 114 patients comparing primary APLS with secondary disease, the two groups were found to have similar clinical and laboratory manifestations of APS. ${ }^{5}$

The patient is treated with lifelong warfarin, with a target INR of 2.0 to 3.0. Anticoagulation is the mainstay treatment for antiphospholipid syndrome. Based on a few studies, the optimum target INR is at a conventional range of 2.0 to 3.0 .6 In patients with recurrent thrombosis despite conventional doses of warfarin, the target INR will be higher at 3.0-4.0 based on expert recommendations, and the effect of adding aspirin to warfarin may increase the frequency of bleeding. However, there is no randomized controlled trial to point towards the effectiveness of increasing INR in recurrent thrombosis. ${ }^{7}$ The duration of anticoagulation is also another issue. The optimum duration of anticoagulation after the first event of venous thromboembolism is debatable. It has been shown that the presence of anticardiolipin antibodies at the end of six months of treatment was associated with a doubling of the risk of recurrent thrombosis (29 versus 14 percent). ${ }^{8}$ Another study has shown that patients with venous thromboembolic episode and antiphospholipid antibodies have an extremely high risk for recurrent venous thrombosis after withdrawal of treatment with oral anticoagulants. ${ }^{9}$ Therefore, based on these recommendations, lifelong anticoagulation is the preferred choice.

\section{REFERENCES}

1. Miyakis S, Lockshin MD, Atsumi T, et al. In ternational consensus statement on an update of the classification criteria for definite antiphospholipid syndrome. J Thromb Haemost 2006; 4(2): 295-306.

2. Martinuzzo ME, Forastiero RR, Carreras LO. Anti b1 glycoprotein I antibodies detection and association with thrombosis. $\mathrm{Br} \mathrm{J}$ Hae matol 1995; 89: 397-402.

3. Kaul M, Erkan D, Sammaritano L, Lockshin MD. Assessment of the 2006 revised an tiphospholipid syndrome classification crite ria. Ann Rheum Dis 2007; 66(7): 927-30.

4. Wilson WA, Gharavi AE, Koike T, Lockshin MD, Branch DW. International consensus statement on preliminary classification cri teria for definite antiphospholipid syn drome: report of an international workshop. Arthritis Rheum 1999; 42(7): 1309-11.

5. Vianna JL, Khamashta MA, Ordi-Ros J, et al. Comparison of the primary and secondary antiphospholipid syndrome: a European Multicenter Study of 114 patients.

Am J Med 1994; 96(1): 3-9.
6. Crowther MA. A comparison of two intensities of warfarin for the prevention of recurrent thrombosis in patients with the an tiphospholipid syndrome. N Engl J Med 2003; 349: 11333-1138.

7. Khamashta MA, Cuadrado MJ, Mujic F, Taub NA, Hunt BJ, Hughes GR. The management of thrombosis in the antiphospholipid-antibody syndrome. N Engl J Med 1995; 332: 993-997.

8. Ruiz-Irastorza G, Hunt BJ, Khamashta MA. A systematic review of secondary thrombopro phylaxis in patients with antiphospholipid antibodies. Arthritis Rheum 2007; 57:1487.

9. Derksen RH, de Groot PG, Kater L, Nieuwen huis HK. Patients with antiphospholipid an tibodies and venous thrombosis should receive long term anticoagulant treatment. Ann Rheum Dis 1993; 52: 689-692. 
